\title{
Sudden death in demyelinating disorders
}

\begin{abstract}
Common cause of death in demyelinating disorders such as Multiple sclerosis has been reported to be infections or cardiorespiratory causes. We report 2 patients who were being investigated for Multiple sclerosis and related disorders who had an unexpected sudden death. Our first case is a 26 year old who presented with Optic Neuritis and past history suggestive of Transverse Mellitus and our second case was a 30 year old woman presented with recurrent Optic Neuritis. They were both being assessed for demyelinating disorders and had sudden unexpected death. More research needs to be done to correlate death directly related to demyelinating diseases.
\end{abstract}

Volume 3 Issue 6 - 2015

\author{
Joseph JP, Rafia H \\ Department of Neurology, Hospital Kuala Lumpur, Malaysia
}

Correspondence: Joseph JP, Department of Neurology, Hospital Kuala Lumpur, Malaysia, Tel 60I22289344, Email jpimy@yahoo.com

Received: October 13, 2015 | Published: December 23, 2015

Keywords: Demyelinating diseases, Medullary lesions

\section{Background}

Common cause of death in demyelinating disorders such as Multiple sclerosis has been reported to be due to complications associated with the background illness. We report two patients who were being investigated for Multiple sclerosis and related disorders that had unexpected sudden deaths.

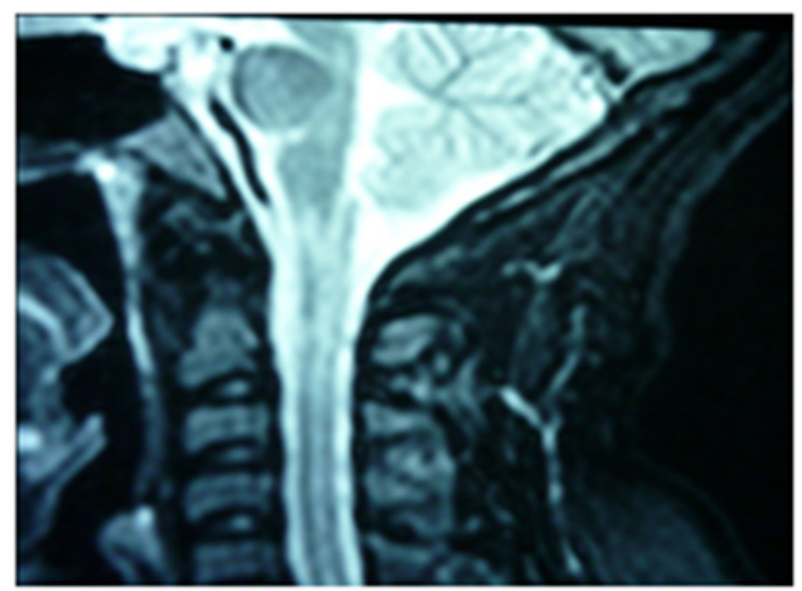

Figure I T2 weighted hyperintense lesions in upper cervical region.

\section{Case I}

A 26-year-old woman had 2 episodes of Right Optic Neuritis 1 year apart where there was a partial resolution after intravenous steroids. She had 1 episode of tetraplegia, which improved after 2 months without any treatment. Clinically, there was evidence of Right Optic atrophy and no other cranial nerve deficit. Left hemiparesis with upper motor neuron deficit was noted and the right upper and lower limbs were normal. No features of connective tissue disease were seen clinically nor were there and blood investigations supporting any other autoimmune disease. Anti-Aquaporin 4 antibody was negative. MRI Brain on February 2012 was normal. However, MRI Spine showed patchy intramedullary lesion extending from C5-T2 seen in central and dorsal aspect. A Repeat MRI done in February 2013 revealed high signal intensity in right corpus callosum, thalamus, corona radiata, lentiform nucleus, insula, cerebral peduncle. Similar lesions were seen in midbrain, medulla oblongata Figure 1. A diagnosis of Multiple sclerosis and a differential of Neuromyelitis Optica Spectrum of Disorder were being considered. She was scheduled a clinic appointment to discuss diagnosis and treatment options. No treatment had been commenced yet. However, she developed sudden onset of shortness of breath while at home and died in the ambulance on the way to the hospital. As the patient was not seen by any physician there were no formal clinical assesment and diagnosis were given for her symptom.

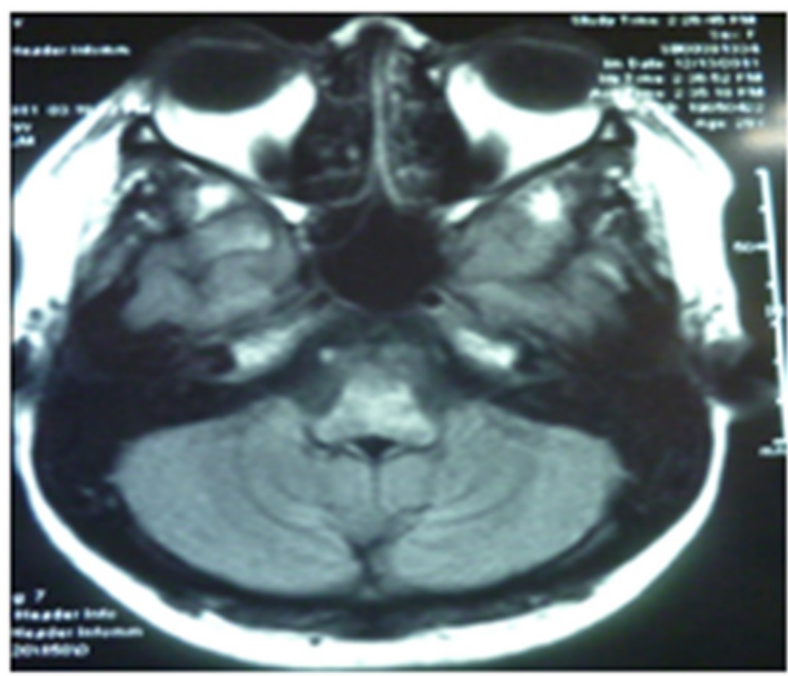

Figure 2 T2 weighted hyperintense lesions in Brainstem region.

\section{Case 2}

Our second case is a 30 -year-old woman who had a history of tetraparesis in 2010. She improved with minimal residual neurological deficit. In August 2011 she presented with Left Hemiparesis and was investigated for young stroke. In November 2011, she presented with Right Optic Neuritis. Clinical examination revealed evidence of Right Optic Neuritis, tone was increased all 4 limbs, power was 4 to $4+$ both upper and lower limbs, reflexes was brisk and Babinski sign was positive over the left. There was also evidence of peripheral asymmetrical sensorimotor neuropathy. All connective tissue screen and Anti-Aquaporin 4 antibody was negative. There were no other evidence of auto-immune disease. MRI showed high signal intensity in right corona radiata, right thalamus, genu of corpus callosum, subcortical white matter, both insula, midbrain and medulla oblongata. Figure 2 cervical spines revealed ill-defined signal intensity at C6 level with no contrast enhancement. Enhancement was seen in both Optic nerves in MRI of the orbits. Oligoclonal band was not sent from the previous hospital where she was managed. She was scheduled for a repeat MRI. Our working diagnosis was Multiple sclerosis with a 
differential diagnosis of Neuromyelitis Opitica Spectrum of Disorder. We could not associate her peripheral neuropathy to any other disorder. She developed sudden onset of shortness of breath and was admitted to a hospital closer to her residence. The patient developed cardiorespiratory arrest and died after a failed resuscitation. A definite cause of death was not known and a post-mortem was not conducted.

\section{Discussion}

Both our patients who were being investigated for demyelinating disorders had medullary lesions, died after shortness of breath. We do not have autopsy reports or radiological evidence to suggest an acute relapse in the brainstem region as death was sudden in onset. However, they had previous Medullary and high Cervical cord lesions. Common cause of death in MS has been attributed to infections or complications related to Multiple sclerosis. Cardiovascular dysfunction related to autonomic neuropathy has been associated with death in MS. ${ }^{1-3}$ Our patients had sudden onset of shortness of breath which could be related to either respiratory or cardiovascular system..$^{4-5}$ Topographic lesions in sudden death in Multiple sclerosis and Neuromyelitis Optica, ${ }^{6}$ have revealed predominant brain stem lesions in one study, and death could also occur in a patient with underlying chronic lesions. ${ }^{7}$ More studies and research are required to establish Medullary lesions causing death in Multiple sclerosis and related disorders. Both are our cases have chronic brainstem lesions and their sudden death could be attributed them. Unfortunately, we could not confirm an acute relapse radiologically or histopathologically due to sudden onset and deterioration of symptoms. There are few case reports and clinical papers associated with death in demyelinating disorders. Therefore, we hope more research are done to ascertain cause of death in such disorders.

\section{Acknowledgments}

None.

\section{Conflicts of interest}

None.

\section{References}

1. Smestad C, Sandvik L, Celius EG. Excess mortality and cause of death in a cohort of Norwegian multiple sclerosis patients. Mult Scler. 2009; 15(11):1263-1270.

2. Grytten Torkildsen N, Lie SA, et al. Survival and cause of death in multiple sclerosis:results froma 50-year follow-up in Western Norway. Mult Scler. 2008;14(9):1191-1198.

3. Sumelahti ML, Tienari PJ, Wikström J, et al. Survival of multiple sclerosis in Findland between 1964 and 1993. Mult Scler. 2002;8(4):350-355.

4. Acevedo AR, Nava C, Arriada N, et al. Cardiovascular dysfunction in multiple sclerosis. Acta Neurol Scan. 2000;101(2):85-88.

5. Drouin E, Nataf S, Lande S, et al. Abnormalities of cardiac repolarization in multiple sclero- sis: relationship with a model of allergic encephalomyelitis in rat. Muscle Nerve. 1998;21(7):940-942.

6. Hengstman GJ, Kusters B. Sudden cardiac death in multiple sclerosis caused by active demyelination of the medulla oblongata. Mult Scler. 2011;17(9):1146-1148.

7. Zhuo L, Zhu S, Sun T, et al. Sudden death of neuromyelitis optica: A case report of clinical missed diagnosis. Rom J Leg Med. 2012;20(4):247250 . 